\title{
Comparison of Two Semi-Empirical BRDF algorithms using SPOT/VGT
}

\author{
Chang Suk Lee and Kyung-Soo Han
}

Department of Spatial information Engineering, Pukyong National University

\begin{abstract}
The Bidirectional Reflectance Distribution (BRD) effect is critical to interpret the surface information using remotely sensed data. This effect was caused by geometric relationship between sensor, target and solar that is inevitable effect for data of optical sensor. To remove the BRD effect, semi-empirical BRDF models are widely used. It is faster to calculate than physical models and demanded less observation than empirical models. In this study, Ross-Li kernel and Roujean kernel were used respectively in National Aeronautics and Space Administration (NASA) and European Organization for the Exploitation of Meteorological Satellites (EUMETSAT) that are used to compare each other. The semi-empirical model consists of three parts which are isotropic, geometric and volumetric scattering. Each part contained physical kernel and empirical coefficients that were calculated by statistical method. Red and NIR channel of SPOT/ VEGETATION product were used to compute Nadir BRDF Adjusted Reflectance (NBAR) over East Asia area from January 2009 to December 2009. S1 product was provided by VITO that was conducted atmospheric correction using Simplified Method of Atmospheric Correction (SMAC). NBAR was calculated using corrected reflectance of red and NIR. Previous study has revealed that Roujean geometric kernel had unphysical values in large zenith angles. We extracted empirical coefficients in three parts and normalized reflectance to compare both BRDF models. Two points located forest in Korea peninsular and bare land in Gobi desert were selected for comparison. As results of time series analysis, both models showed similar reflectance change pattern and reasonable values. Whereas in case of empirical coefficients comparison, different changes pattern of values were showed in isotropic coefficients.
\end{abstract}

Key Words : BRDF, SPOT/VGT, surface reflectance

\section{Introduction}

The Bi-directional Reflectance Distribution (BRD) effect was caused by strong anisotropic reflectance of surface that was inevitable phenomenon for space-born optical sensor. It caused in various conditions showed an ingredient of noise like signal for a long time surface observation. Since BRD effect is one of significant error source to interpret surface information such as surface reflectance, albedo and vegetation indices, it

Received June 11, 2013; Revised June 16, 2013; Accepted June 17, 2013.

'Corresponding Author: Kyung-Soo Han (kyung-soo.han@pknu.ac.kr)

This is an Open-Access article distributed under the terms of the Creative Commons Attribution Non-Commercial License (http:/creativecommons.org/ licenses/ by-nc/3.0) which permits unrestricted non-commercial use, distribution, and reproduction in any medium, provided the original work is properly cited 
must be removed to retrieval surface properties. The Bi-directional Reflectance Distribution Function (BRDF) is also an important source of biophysical information about land surface observation and determined by land surface structure and optical characteristics (Lutch et al., 2000).

BRDF model corrects these fluctuations that were divided into three groups: physical, empirical and semiempirical models (Wanner et al., 1995). The physical models physically simulated the scattering of light explicitly. But it takes a lot of time to calculate and there is no model that can be applied to all land cover. Whereas empirical model, perform the calculation through the empirical relationship of the observations than simulated statistically. It needs various angular circumstance of observation to correct BRD effect. In fact, remotely sensed data by the optical sensor onboard the satellite has several problems such as cloud cover, orbit and instrument characteristics (Wanner $e t$ al., 1995). Lastly, semi-empirical model, it used combination of physical kernels and empirical coefficients. Calculating process of semi-empirical model is simpler than physical and needed fewer number of observations than empirical model. Furthermore, semi-empirical model is easy to invert for calculating albedo using analytical solution (Lucht $e t$ al., 2000).

There are two semi-empirical models: Ross-Li
(Wanner et al., 1995) and Roujean (Roujean et al., 1992). These models were used for operational purpose in National Aeronautics and Space Administration (NASA) and European Organization for the Exploitation of Meteorological Satellites (EUMETSAT). In this study, we had compared kernels and empirical coefficients of two semi-empirical models and examined uncertainty about each model. The uncertainty of each model is important to estimation albedo, which is one of the important parameters in global climate and weather models (Pokrovsky and Roujean, 2002).

\section{Data and Study area}

The Satellite Pour I'Obervation de la Terre (SPOT)/ VEGETATION were developed for participating to the international improvement of studies of the biosphere, especially in vegetation (Saint, 1994). It provided daily global coverage and has $1 \mathrm{~km}$ spatial resolution. Several products of SPOT/VGT have been distributed free for research purpose through Vision on TechnOlogy (VITO, http://free.vgt.vito.be/) that can be divided into two kinds such as $\mathrm{P}$ and $\mathrm{S}$ products. The $\mathrm{P}$ product contains apparent reflectance at the Top Of Atmosphere (TOA), and the S products include S1 and S10 are Maximum Value Composite (MVC) or synthesis data. In this study, daily synthesis (S1) product was synthesized

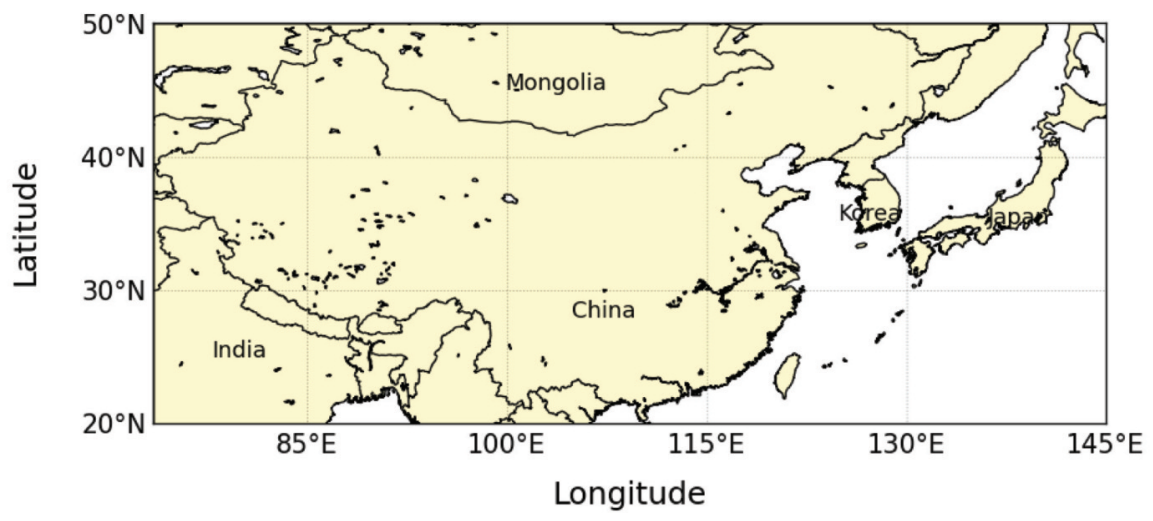

Fig. 1. Study area in East Asia. 
only takes into account multiple measurements obtained through the overlap between successive passes at high latitudes (Verbeiren, 2009), was used for normalized surface reflectance. In cast of S10 product, it inappropriate for BRDF modeling because of many angular data was filtered by MVC method. The $\mathrm{S}$ product was carried out radiometric, geometric correction and atmospheric effects were also corrected by Simplified Method Atmospheric Correction (SMAC) which was developed by Rahman and Dedieu (1994). The $\mathrm{S} 1$ product consists of each band reflectance values and angular information and atmospheric condition data. Among the four SPOT-VGT bands, B2 (Red, $0.61-0.68 \mu \mathrm{m}$ ) and B3 (Near Infrared, $0.79-0.89 \mu \mathrm{m})$ bands which are represented well the characteristic of the vegetation were used for BRDF modeling.

Fig. 1 shows study area in this study which included the entire continent of East Asia, including Korea, Japan, and China. This area has various land types and also includes two broad regions of dessert (Gobi and Taklamakan). In many previous studies, this region was dealt with the area of interest for vegetation, land cover and climatic change (Pi et al., 2009; Kim et al., 2011; Park et al., 2012).

\section{Method}

There are three kinds of BRDF models: physical, empirical and semi-empirical. The physical model is complex and precise due to fully mathematically simulated BRD effect at homogeneous surface. Therefore, physical model had some constraints. First is computational complexity that caused long running time and complicated inversion processes. Second, actual land surface is almost heterogeneous for middle/low resolution optical sensors. Furthermore, physical model optimize certain land cover type thus, it is difficult to apply for every land cover type. The empirical model required many measurements corresponding to various angular condition (zenith and azimuth angle of solar and sensor) because it is retrieved based on many observations by statistical method. In case of remote sensing using satellite, the number of observations in any given time period is restricted by orbital and instrument property and atmospheric condition (Wanner et al., 1995). The semiempirical model combined physical kernel and with empirical coefficients. It required less observation than empirical and much simpler than physical model and can be applied various land cover type. Above all it can be inverted analytically and rapidly through analytical approach (Wanner et al., 1995; Hu et al., 1997; Roujean et al., 1997). So we selected semi-empirical approach for this study. The semi-empirical BRDF model generally has followed specific form which was suggested by Roujean et al (1992).

$$
\mathrm{R}\left(\theta_{s}, \theta_{v}, \Phi\right)=K_{0}+K_{1} f_{1}\left(\theta_{s}, \theta_{v}, \Phi\right)+K_{2} f_{2}\left(\theta_{s}, \theta_{v}, \Phi\right)
$$

In equation (1), $\theta_{s}$, and $\theta_{v}$ are solar and satellite zenith angle respectively, and $\Phi$ is relative azimuth angle between sun and sensor. The $f_{1}$ and $f_{2}$ are physical kernels that means geometric and volumetric scattering respectively. These kernels are function of view zenith and illumination zenith and relative azimuth angle that describes the surface scattering from canopy (Geo et al, 2000). $K_{(0,1,2)}$ are empirical coefficients ( $K_{0}$-isotropic, $K_{1}$ geometric, $K_{2}$-volumetric) that characterized surface BRD properties (Roujean et al., 1992).

In semi-empirical approach, measurement reflectance by satellite is assumed to consist of isotropic, geometric, and volumetric scattering in semi-empirical BRDF model. Surface reflectance was determined by sum of the three scattering type. Each scattering reflectance was represented as the product kernel and coefficient which controlled physical BRD value from kernel. Thus semi-empirical BRDF can apply to various land type.

There are two semi-empirical BRDF models (Roujean and Ross-Li) for operational purpose, these 


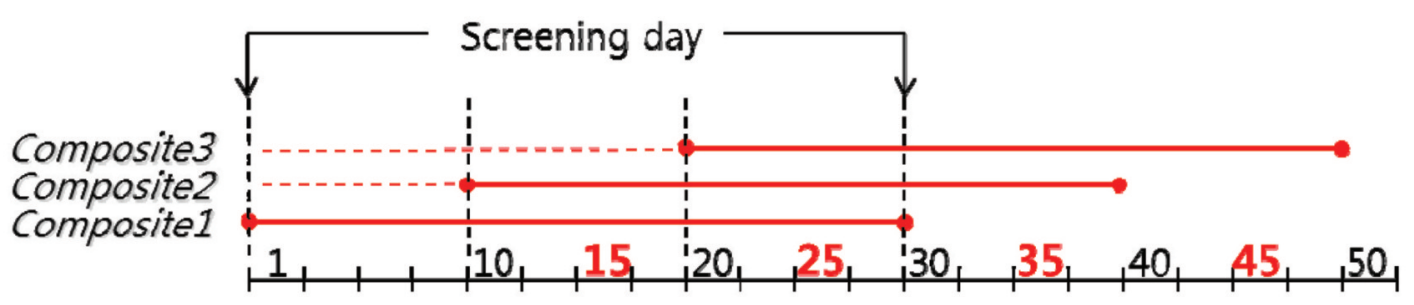

Fig. 2. Concept of synthesis for normalized surface reflectance.

are employed by EUMETSAT and NASA respectively. To calculate nadir BRDF-adjusted reflectance, process of normalization of directional effects is essential. In this paper, we introduced additive method which was proposed by Duchemin and Maisongrande (2002).

$$
\begin{gathered}
\rho_{\text {norm }}=\rho_{\text {model }}\left(\theta_{s}=\text { mean } \theta_{s}, \theta_{v}=0^{\circ}\right) \\
+\rho_{\text {measured }}-\rho_{\text {model }}\left(\theta_{s}, \theta_{v}, \Phi\right) \\
\rho_{N}=\frac{1}{\mathrm{n}} \sum_{i=1}^{n} \rho_{\text {norm }}(i)
\end{gathered}
$$

Each term in equation (2) and (3) means following description:

$\rho_{\text {norm }}$ - Normalized surface reflectance

$\rho_{\text {model }}\left(\theta_{s}=\operatorname{mean} \theta_{s}, \theta_{v}=0^{\circ}\right)$ - Adjusted reflectance at nadir viewing and mean solar zenith angle during synthesis period

$\rho_{\text {measured }}$ - Surface reflectance measured by satellite

$\rho_{\text {model }}\left(\theta_{s}, \theta_{v}, \Phi\right)$ - Calculated surface reflectance using kernels with yielded empirical coefficients

$\rho_{N}$ - Synthesized normalization of surface reflectance

$\mathrm{n}$ - The number of cloud free day within synthesis period

The above-mentioned method was validated by Duchemin et al. (2002) through comparison with MVC methods. It is required various zenith and azimuth angle for BRDF model smoothly, thus suitable synthetic period setting is import. Representative adjusted surface reflectance was produced as 10-day and 16-day synthesis for SPOT/VGT and Terra/MODIS, respectively, because these products have constraint for a real-time purpose (Duchemin and Maisongrande, 2002).

SPOT/VGT has 26-day orbital cycle with a 5-day sub-cycle (Duchemin and Maisongrande, 2002;
Verbeiren, 2009). Leroy et al. (1997) have synthesized 30-day windows with a 10-day interval for POLDER/ ADEOS. Duchemin and Maisongrande (2002) showed long screening is more efficient than short screening. The process of normalization was set-up 30-day time windows and 10-day interval for monthly representative of surface reflectance in this study. Fig. 2 briefly showed that concept of normalization process.

We compared two different semi-empirical models and carried out kernel and empirical coefficients analysis.

\section{Results \& Discussion}

\section{1) Kernel analysis}

Roujean's BRDF and Ross-Li BRDF use same volumetric kernel and different geometric kernel. The

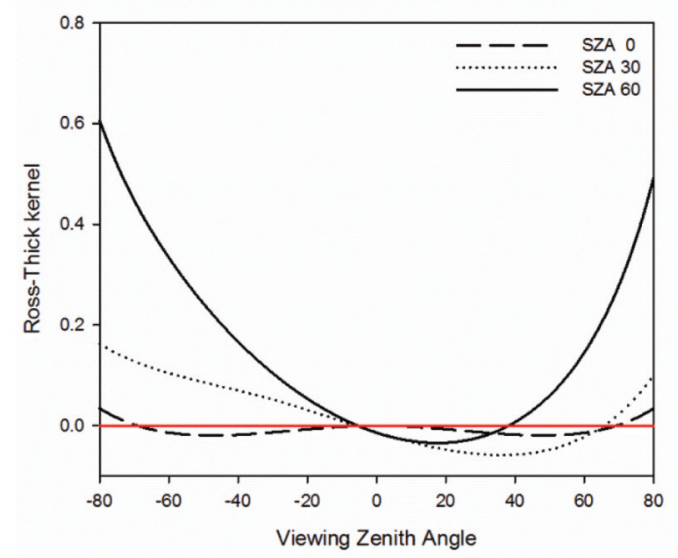

Fig. 3. Values of Ross-thick kernel for three solar zenith angle $\left(0^{\circ}, 30^{\circ}, 60^{\circ}\right)$ on principal plane. 
volumetric kernel is based on radiative transfer theory, which is a single scattering approximation in homogeneous medium plant canopies that was started out from a formula taken from Ross (1981) (Roujean et al., 1992; Wanner et al., 1995; Lucht and Roujean, 2000). Socalled Ross-thick kernel is the following equation (4).

$$
f_{v o l}\left(\theta_{s}, \theta_{v}, \Phi\right)=\frac{4}{3} \frac{1}{\cos \theta_{s}+\cos \theta_{v}} \times\left[\left(\frac{\pi}{2-\xi}\right) \cos \xi+\sin \xi\right]-\frac{1}{3}
$$

The $\xi$ is the phase angle of scattering, and $\cos \xi$ is calculated by following equation (5)

$$
\cos \xi=\cos \theta_{s} \cos \theta_{v}+\sin \theta_{s} \sin \theta_{v} \cos \Phi
$$

The Ross-thick kernel performed bowl-shape corresponding to change viewing and solar zenith angle (Fig. 3).

First, Roujean's geometric kernel has following equation.

$$
\begin{aligned}
& f_{\text {geo }}\left(\theta_{s}, \theta_{v}, \Phi\right)=\frac{1}{2 \pi}[(\pi-\Phi) \cos \Phi+\sin \Phi] \tan \theta_{s} \tan \theta_{v} \\
& -\frac{1}{2 \pi}\left(\tan \theta_{s}+\tan \theta_{v}+\sqrt{\tan ^{2} \theta_{s}+\tan ^{2} \theta_{v}-2 \tan \theta_{s} \tan \theta_{v} \cos \Phi}\right)
\end{aligned}
$$

Roujean et al. (1992) had suggested this kernel which assumed that the sub-pixel randomly contains a large number of protrusions on Lambertian surface and shadowed areas by protrusions are absolutely dark and mutual shadow between protrusions can be neglected.

Second, Li-Sparse geometric kernel has following equation.
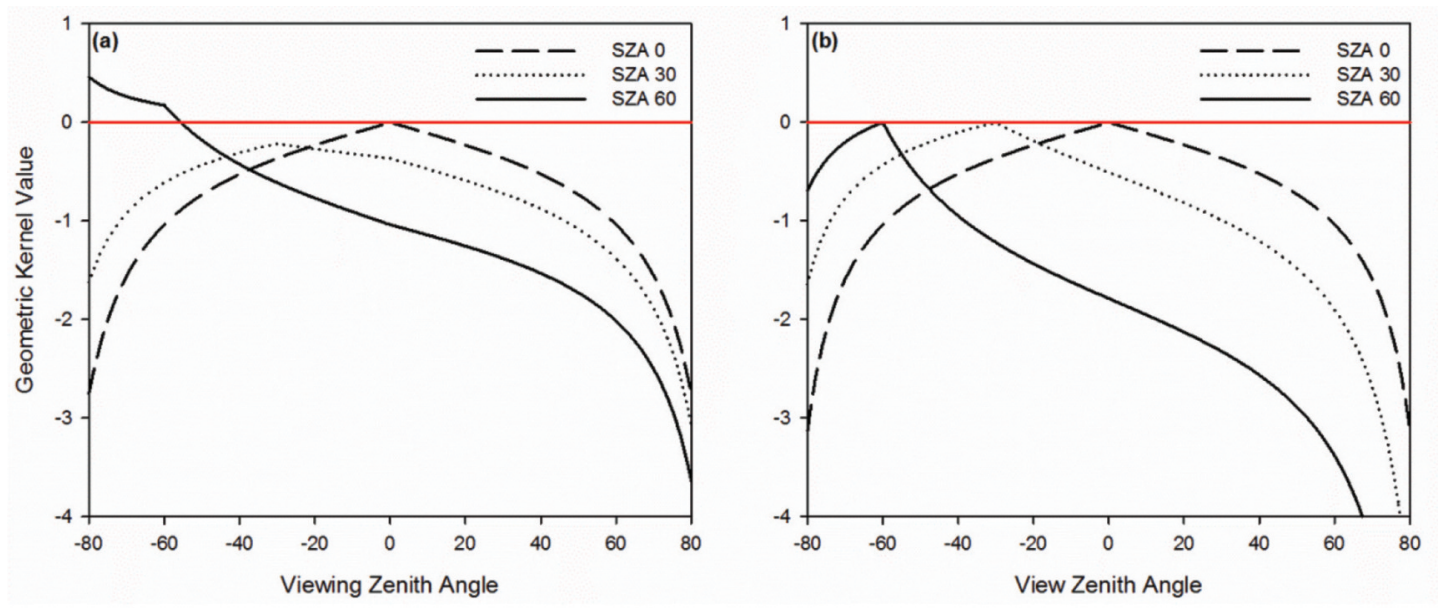

Fig. 4. Values of geometric kernel (a: Roujean, b: Li-Sparse) for three solar zenith angle $\left(0^{\circ}, 30^{\circ}, 60^{\circ}\right)$ on principal plane. 

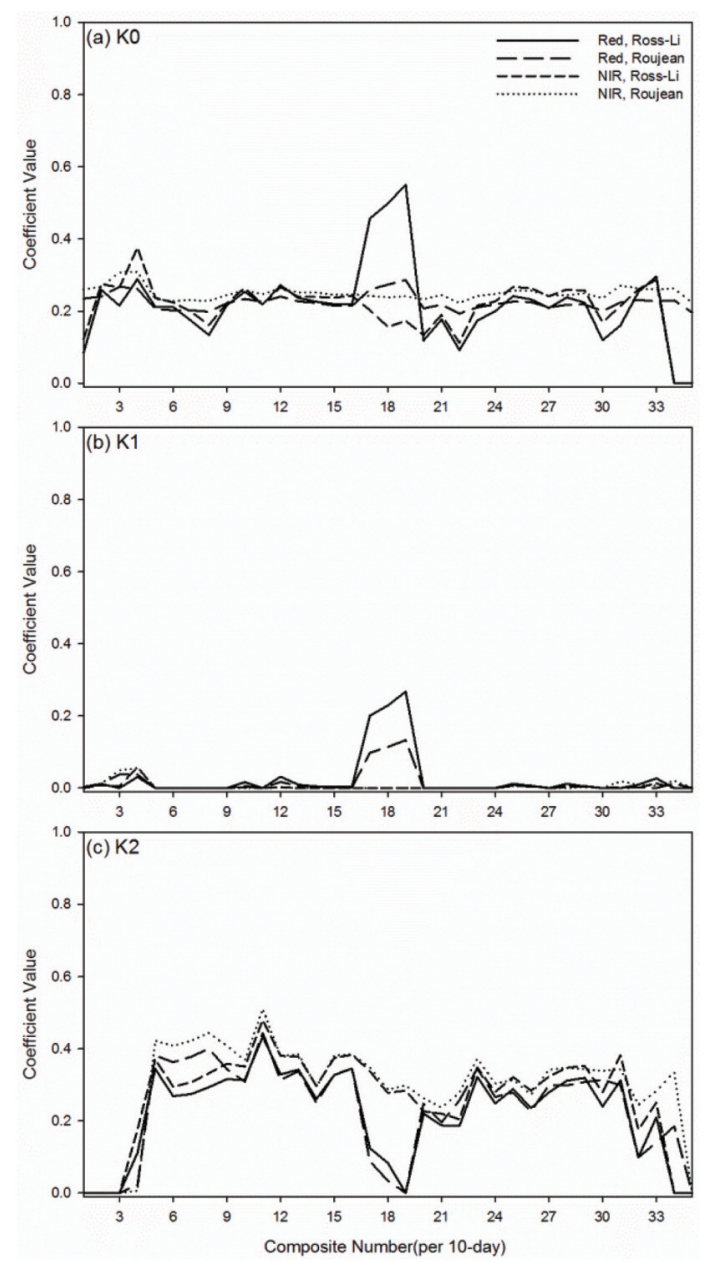

Fig. 5. Time series analysis of three coefficients (a: KO- isotropic, b: K1-geometric, c:K2-volumetric) on barren land.

\section{2) Empirical coefficients analysis}

Calculated empirical coefficients for red, NIR bands by BRDF model were analyzed on two land-cover types (mixed forest, barren land) during one year (2007). Two points were selected to comparison of coefficients analysis according to MODIS Land Cover product. In order to confirm contrastable reflectance property corresponding to land cover type, we selected two points that one point is mixed forest in Korea peninsula another point is barren land in Taklamakan dessert. All coefficients for both BRDF models showed similar pattern of change value for each band on both land-cover types (Fig. 5 and Fig. 6). Generally in time
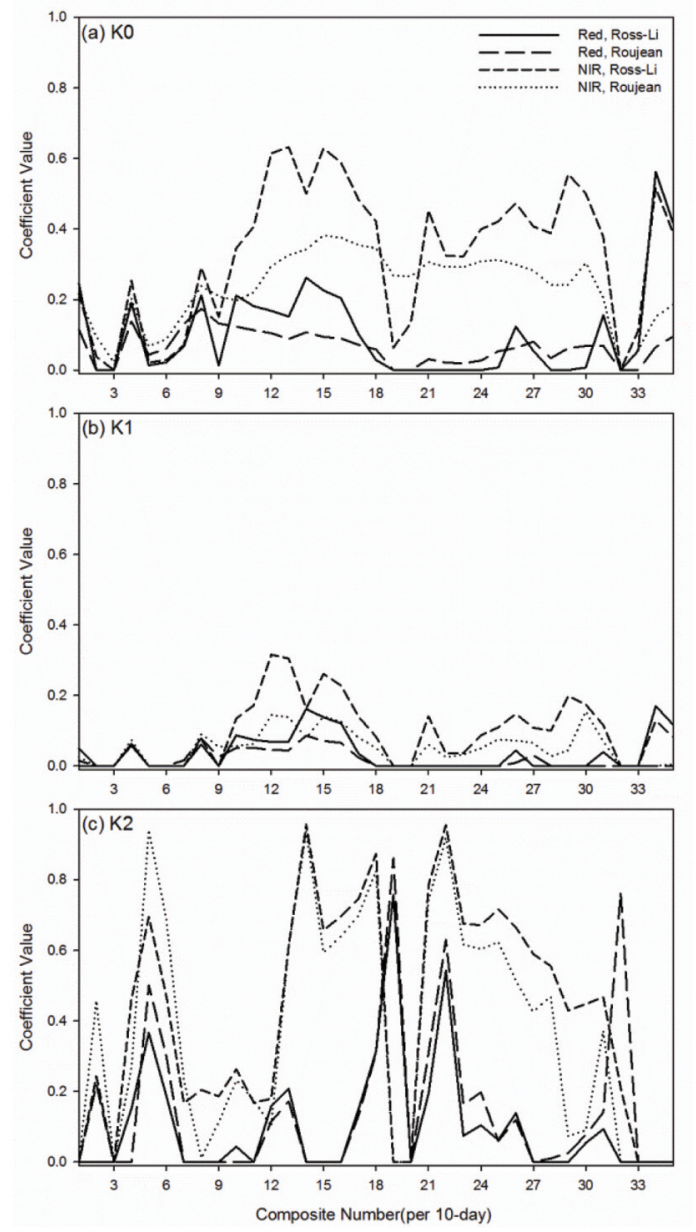

Fig. 6. Time series analysis of three coefficients (a: KO- isotropic, b: K1-geometric, c:K2-volumetric) on mixed forest.

series analysis, magnitude of change of coefficients in Ross-Li BRDF model larger than Roujean BRDF model. Among the tree coefficients, $K_{0}$ has largest different between two kernels. Fig. 7 showed that normalized reflectance by two BRDF model change pattern on both land cover types. Both BRDF models showed that reasonable result according to each land cover type. The $K_{0}$ should be represent normalized surface reflectance since it is isotropic coefficient. When compares with time series analysis of coefficient and reflectance, $K_{0}$ of Ross-Li model had different change pattern. For red band, $K_{0}$ had considerable high peak during summer in barren land. In case of NIR band, dramatic change during growth of vegetation 

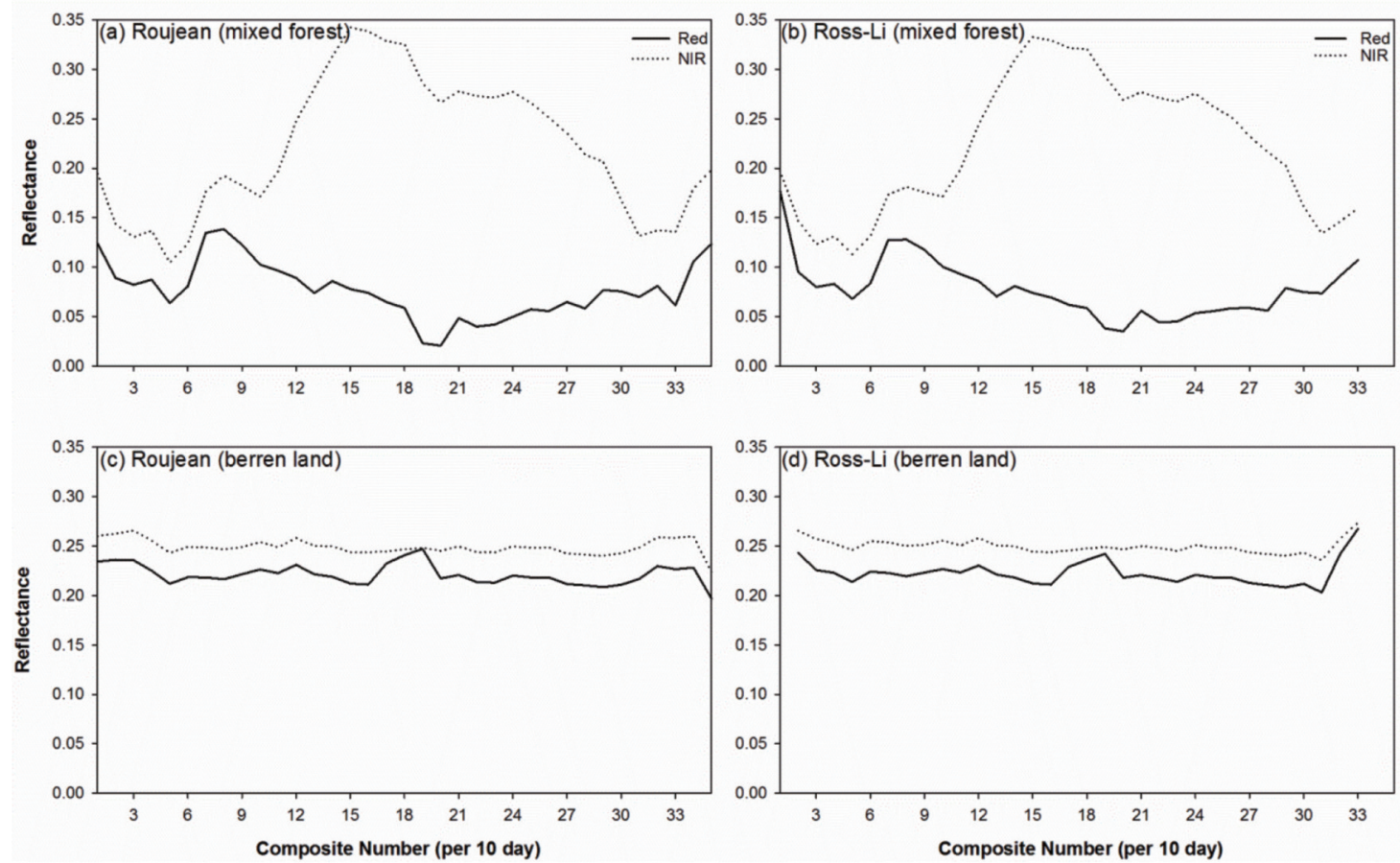

Fig. 7. Normalized surface reflectance using each BRDF model (a and c : Roujean model, $b$ and $d$ : Ross-Li model) corresponding to two land cover types (solid line: red band, dot line: NIR band).

season had been observed. In summer, underestimation trend appeared, whereas overestimation trend appeared in spring and autumn.

\section{Conclusions}

We had examined change pattern of kernel and empirical coefficients. Result of several analyses, Roujean geometric kernel has unphysical behavior in high zenith angle, on the other hand, Ross-Li BRDF model has unreasonable change pattern in $K_{0}$. The BRDF modeling is essential to calculate albedo through inversion process which were used each kernel and empirical coefficients. As a result of analysis in section 4, two BRDF models have some uncertainty. User of BRDF model should be aware of these points to carry out BRDF modeling.

\section{Acknowledgment}

This work was funded by the Korea Meteorological Administration Research and Development Program under Grant CATER 2012-2066.

\section{References}

Duchemin, B., and P. Maisongrande, 2002. Normalization of directional effects in 10-day global syntheses derivedfrom VEGETATION/SPOT: 1.Investigation of concepts based on simulation, Remote Sensing of Environment, 81: 90-100.

Duchemin, B., B. Berthelot, G. Dedieu, M. Leroy, and P. Maisongrande, 2002. Normalization of directional effects in 10-day global syntheses derived from VEGETATION/SPOT: 2. Validation of an operational method on actual data sets, Remote Sensing of Environment, 81: 101-113. 
Kim, S.I., K.-S. Han, K.-J. Pi, 2011. The Trend Analysis of Vegetation Change Applied to Unsupervised Classification Over East Asia: Using the NDVI 10-day data in 1999 2010, The Korean Society for Geo Spatial Information System, 19(4): 153-159 (in Korean with English abstract).

Geo, F., X. Li, A. Strahler, and C. Schaaf, 2000. Evaluation of the Li transit kernel for BRDF modeling. Remote Sensing Reviews, 19(1-4): 205-224.

Hu, B., W. Lucht, X. Li, and A.H. Strahler, 1997. Validation of Kernel-Driven Semiempirical Models for the Surface Bidirectional Reflectance Distribution Function of Land Surfaces, Remote Sensing of Environment, 62: 201-214.

Lucht, W., C.B. Schaaf, A.H. Strahler, 2000, An algorithm for the retrieval of albedo from space using semiempirical BRDF models, IEEE Transactions on Geoscience and Remote Sensing, 38(2): 977-998.

Lucht, W., and J.-L. Roujean, 2000. Considerations in the Parametric Modeling of BRDF and Albedo from Multiangular Satellite Sensor Observations, Remote Sensing Reviews, 18: 343-379.

Rahman, H., and G. Dedieu, 1994. SMAC: a simplified method for the atmospheric correction of satellite measurements in the solar spectrum. International Journal of Remote Sensing, 15(1): 123-143.

Ross, J.K., 1981. The Radiation Regime and Architecture of Plaint Stands, Dr. W. Junk Publishers, The Hague, p. 392.

Roujean, J.L., D. Tanré, F.-M. Bréon, and J.-L. Deuzé, 1997. Retrieval of land surface parameters from airborne POLDER bidirectional reflectance distribution function during HAPEX-Sahel, Journal of Geophysical Research, 97(D10): 11201-11218.

Roujean, J.L., M. Leroy, and P.Y. Deschamps, 1992. A bidirectional reflectance model of the Earth's surface for the correction of remote sensing data, Journal of Geophysical Research, 97 (D18): 20455-20468.

Park, E.-B., K.-S. Han, C.-S. Lee, and K.-J. Pi, 2012. The Tendency Analysis of Albedo by Land Cover Over Northeast Asia Using MODIS 16Day Composited Albedo data, Korean Journal of Remote Sensing, 28(5): 501-508 (in Korean with English abstract).

Pi, K.-J., K.-S. Han, and S.-J. Park, 2009. A Comparative Analysis of Land Cover Changes Among Different Source Regions of Dust Emission in East Asia: Gobi Desert and Manchuria, Korean Journal of Remote Sensing, 25(2): 175-184 (in Korean with English abstract).

Pokrovsky, O., and J.-L. Roujean, 2002. Land surface albedo retrieval via kernel-based BRDF modeling: 1. Statistical inversion method and model comparison, Remote Sensing of Environment, 84: 100-119.

Saing, G., 1994, "VEGETATION” onboard SPOT 4 MISSION SPECIFICATIONS Version 3, p 32. Verbeiren, S., 2009, GMES Space Component Data Access(GSC-DA) Instrument/Product specifications, VITO, p42.

Wanner, W., X. Li and A.H. Strahler, 1995. On the derivation of kernels for kernel-driven models of bidirectional reflectance. Journal of Geophysical Research, 100(D10): 2107721089. 